\title{
Deployment and Evaluation of a Real-time Kinematic System Using tinc-VPN Software
}

\author{
Xing Liu, Tarig Ballal, Martins Bruvelis, and Tareq Y. Al-Naffouri \\ Computer, Electrical and Mathematical Science and Engineering Division \\ King Abdullah University of Science and Technology (KAUST) \\ Thuwal, Saudi Arabia \\ \{xing.liu; tarig.ahmed; martins.bruvelis; tareq.alnaffouri\} @ kaust.edu.sa
}

\begin{abstract}
Real-time kinematic (RTK) is the most popular GNSS-based precise positioning technique that is widely used in many navigation and control systems. RTK is a relative positioning method, which takes advantage of pseudo-range and carrier-phase observations received at a mobile GNSS receiver (rover) and a fixed base station. After fixing the integer ambiguities, the carrier-phase observables can be utilized for precise positioning such that RTK can estimate the rover's position with centimeter-level accuracy. In order to produce instantaneous location information, correction data needs to be effectively transferred from the base station to the rover via a communication link. Various ways of correction information transmission have been proposed over the years. In this work, we utilize the Networked Transport of RTCM via Internet Protocol (NTRIP) for data transmission. We establish the connection between the base station and the rover through a virtual private network (VPN), which allows the rover to communicate with the base station using a local IP address, regardless of the nature of the Internet connection on both sides. To study the impact of the quality of the Internet connection, especially with respect to latency, we evaluate the performance of RTK positioning under different Internet connection conditions. We carry out experiments using commercial GNSS RTK products.
\end{abstract}

Index Terms-GNSS, RTK, Localization, VPN, Network latency

\section{INTRODUCTION}

Real-time kinematic (RTK) is an essential technology for many emerging applications that require accurate position information, such as autonomous outdoor robots and autonomous vehicles [1], [2]. RTK is a positioning technique that is based on carrier-phase ranging, which is orders of magnitude more precise than code-based ranging [3], [4]. RTK is capable of delivering centimeter-level position accuracy of a rover by utilizing correction data received from a GNSS base station deployed at a fixed location [5]. The rover determines its position using techniques that involve ambiguity resolution and differential correction. In order to achieve realtime operation, the correction information has to be received in a timely manner via a communication link between the rover and the base station [6].

The performance of RTK positioning depends, among other things, on the length of the baseline connecting the rover and the base station, the quality of the base station and rover receivers, and the quality of the correction data received by the rover [7]. The accuracy of the correction data is a function of many things that include the quality of the base station observations, which is impacted by effects such as multipath and interference. These effects depend mainly on the base station location, which has to be judiciously selected. Another critical factor that affects RTK positioning performance is the quality of the communication link between the rover and the base station, which impacts the amount of time required to transfer the data from the base station to the rover [8].

Traditional RTK systems utilized low-cost transmission of radio signals, typically in the very high frequency (VHF) or ultra-high frequency (UHF) band [9]. As an alternative approach to correction data transmission, NTRIP has been proposed [8]. NTRIP is a protocol designed for delivering differential GNSS data over the Internet. NTRIP allows for correction data access without relying on bulky RF equipment (an internet SIM might be sufficient). In addition, NTRIP mitigates the effect of RF interference on the transmission of the correction data and facilitates access to multiple base stations without requiring extra hardware. The actual transmission of data using NTRIP can take place over different kinds of networks such as GSM, LTE, GPRS, EDGE, and UMTS [10]. The data transmission can also happen over private networks or mixed networks. Due to the difference in architecture and the unpredictability of these networks, the availability of correction data and the latency associated with the delivery of such data is crucial to the performance of RTK positioning.

In this work, we describe the deployment of a GNSS system for RTK positioning. The deployment consists of a GNSS base station connected to the internet using LTE (4G). The base station and the rover devices are connected through a VPN created using tinc software. Tinc is a free VPN daemon that is able to build a secure private network between hosts on the Internet by employing tunneling and encryption technology. Tinc is licensed under the GNU General Public License version 2 or later. In a tinc VPN, devices are allowed to exchange information with each other over the Internet without exposing any information to others. In this work, a tinc VPN is used in association with NTRIP. The setup tested in this work enables secure connectivity without requiring the base station to have a fixed (public) IP address, which may not be achievable at a reasonable cost in remote outdoor locations.

This contribution is structured as follows. In Section II, we describe the fundamental methodology of RTK positioning, 
including basic knowledge of measurement models, differential techniques, and parameter estimation algorithms. In Section III, we describe the software and hardware components of the system and the characteristics of the employed communication link. In Section IV, we present experimental results of RTK positioning under varying network conditions at the rover side and provide a detailed evaluation of RTK positioning performance.

\section{RTK Positioning Methodology}

RTK takes advantage of two types of observations, i.e., pseudo-range and carrier-phase measurements, which are linearly combined in a double-difference model to eliminate/reduce various errors. Considering a setup with a single rover and a single base station, as shown in Fig. 1, the doubledifference observations can be expressed as

$$
\begin{gathered}
P_{r b}^{n m}=\left(\mathbf{h}_{r}^{n m}\right)^{T} \mathbf{x}_{r b}+I_{r b}^{n m}+T_{r b}^{n m}+\varepsilon_{r b}^{n m}, \\
\varphi_{r b}^{n m}=\left(\mathbf{h}_{r}^{n m}\right)^{T} \mathbf{x}_{r b}+\lambda N_{r b}^{n m}-I_{r b}^{n m}+T_{r b}^{n m}+\xi_{r b}^{n m},
\end{gathered}
$$

where $P_{r b}^{n m}$ is the double-difference pseudo-range measurement, $\varphi_{r b}^{n m}$ is the double-difference carrier-phase observation, the subscripts $r$ and $b$ represent the rover and base station, respectively, the superscripts $n$ and $m$ refer to satellite numbers, $\mathbf{h}_{r}^{n m}$ is the difference between the line-of-sight vectors of satellite $m$ and satellite $n$ as observed from the rover, and $\mathbf{x}_{r b}$ is the baseline vector between the rover and the base station, $N_{r b}^{n m}$ is the unknown integer carrier-phase ambiguity, $I_{r b}^{n m}$ is the remaining ionospheric delay, $T_{r b}^{n m}$ is the remaining tropospheric delay, and the additive noise and unmodeled errors are expressed as $\varepsilon_{r b}^{n m}$ and $\xi_{r b}^{n m}$.

Double differencing can remove satellite clock bias, receiver clock bias, and other systematic errors, yet it may not eliminate atmospheric effects completely. The magnitude of atmospheric residual usually depends on the distance between the rover and reference station, i.e., the baseline length. Generally, for short baselines, the atmospheric delay terms are considerably reduced and can be ignored. However, for long baselines, the magnitude of the atmospheric bias is still too large to be ignored. In this case, more advanced mitigation techniques are required.

Carrier-phase ambiguity resolution is crucial for centimeterlevel RTK accuracy. The convergence time, i.e., the time to fix the integer ambiguities successfully varies depending on different factors such as the baseline length, satellite-receiver geometry, receiver surrounding environment, internet connection quality, and so on. Instantaneous ambiguity resolution (the integer ambiguities can be resolved using a single epoch of measurements) is only feasible for short baselines with multifrequency data. For the short-baseline single-frequency model or the long-baseline model, multi-epoch measurements are required to retrieve the integer ambiguities reliably. Typically, a Kalman filter is used to estimate the unknown parameters as the system states, including position, float carrier-phase ambiguities, residual atmospheric delays, and hardware biases. In this process, real-time data transmission between the rover and

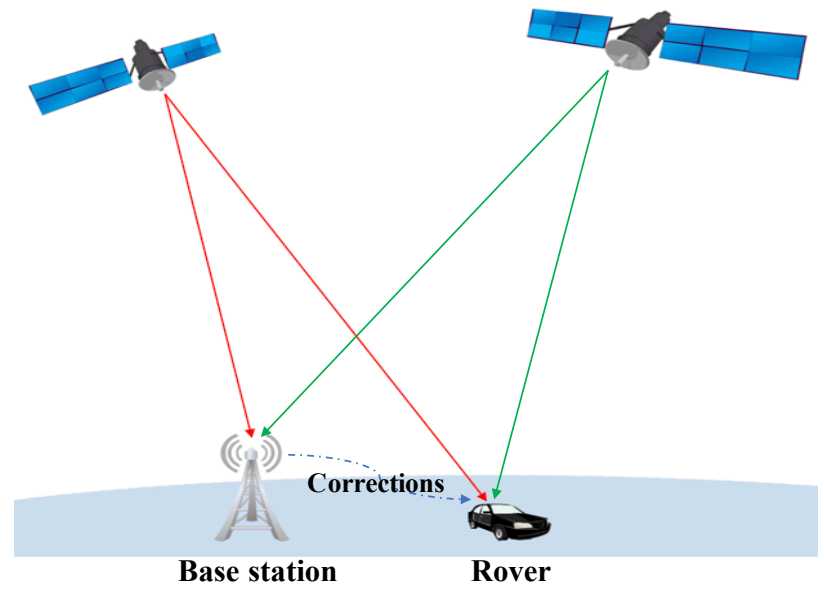

Fig. 1. Illustration of RTK system.

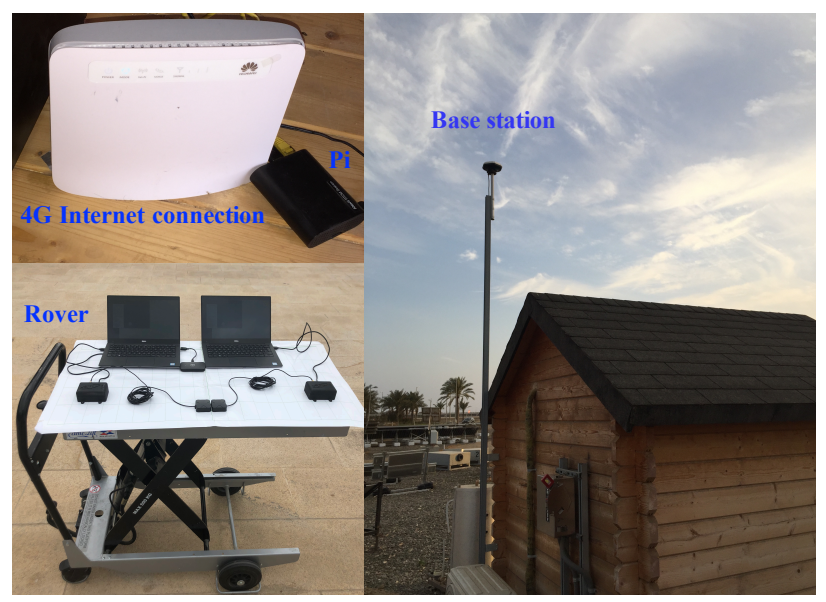

Fig. 2. Deployed RTK system components.

the base station is essential. The float ambiguities will improve over time and can be fixed to the correct integers after a period of refinement. Then the fixed solution is computed based on integer carrier-phase ambiguities. Usually, the position of base station is precisely known. Hence, once we successfully recover the unknown integer carrier-phase ambiguity $N_{r b}^{n m}$, the relative position $\mathbf{x}_{r b}$, and hence the absolute location of the rover can be calculated with high accuracy.

\section{RTK SYSTEM DEPLOYMENT}

This section is devoted to the description of our RTK system deployment. We give a brief description of various system components. Following that, we look into the system operation and the main characteristics of the system.

\section{A. GNSS Base Station}

As shown in Fig. 2, a GNSS base station is firmly fixed at a position with a good view of satellites to reduce the impact of multipath as much as possible. The base station consists of a Raspberry $\mathrm{Pi}$ that is connected to the Internet via a $4 \mathrm{G}$ router. Before the RTK experiment, the base station has been calibrated to estimate its precise absolute position, 
which is completed by running the software installed on a laptop. Different ways, such as Wi-Fi, USB, or Bluetooth, can be used to connect the Raspberry Pi to the laptop. In this process, one can choose to stream correction data from an external base station or use filtered least-squares to obtain a solution with good accuracy. After the calibration process, the Raspberry Pi is configured to run NTRIP-Caster software to stream correction data to the rover automatically. The configured NTRIP-Caster can support three sources at most, and each source is able to deal with up to 10 rovers. In our test, the single-source and single-rover case is considered.

\section{B. The Rover}

For the rover configuration, as depicted in Fig. 2, a GNSS receiver with an external antenna is firmly mounted on the moving platform or the vehicle. The receiver connects to a laptop using a USB cable. The laptop, with network access via a wireless or wired link, will execute the RTK software, which is responsible for data processing. All the devices, whether a laptop or a Raspberry $\mathrm{Pi}$, are running the Ubuntu operating system.

\section{Network Connectivity Using VPN}

The data communication link is necessary for the momentary data transmission (pseudo-range, carrier-phase, and ancillary data) from the base station to the rover. The correction data is transmitted via the Internet using NTRIP to the mobile receiver, wherein RTCM3 data protocols are used.

The connection between the rover and the base station is established through a VPN implemented using tinc software, which is an open-source, self-routing, automatic full mesh networking software and daemon. Fig. 3 shows an illustration of the network connection. A VPN appears to the IP level network code as a standard network device. It can establish a secure private network with different parts of the RTK system on the Internet. Consequently, the reference station data can be transmitted to the rover via the Internet without exposing any information. Since it is easy to expand the VPN using tinc software, a similar configuration can be extended to multi-receiver and multi-base-station mode. Therefore, a secure private network between the laptop, Raspberry Pi, and campus server is available.

Generally, the network quality at the base station (Raspberry $\mathrm{Pi}$ ) can be guaranteed since it is static and firmly fixed at a place that is carefully chosen. In contrast, stable connectivity cannot be guaranteed at the rover location. Hence, we focus on studying the impact of rover network quality on RTK performance.

\section{EXPERIMENTAL RESUlTS AND Discussion}

The deployed RTK system was tested using $4 \mathrm{G}$ and Wi$\mathrm{Fi}$ as Internet connection methods at the rover. Two identical GNSS receivers and laptops were utilized in this experiment. The receivers were mounted on a movable platform close to each other such that their positions are nearly the same. This excludes position as a factor affecting the results obtained

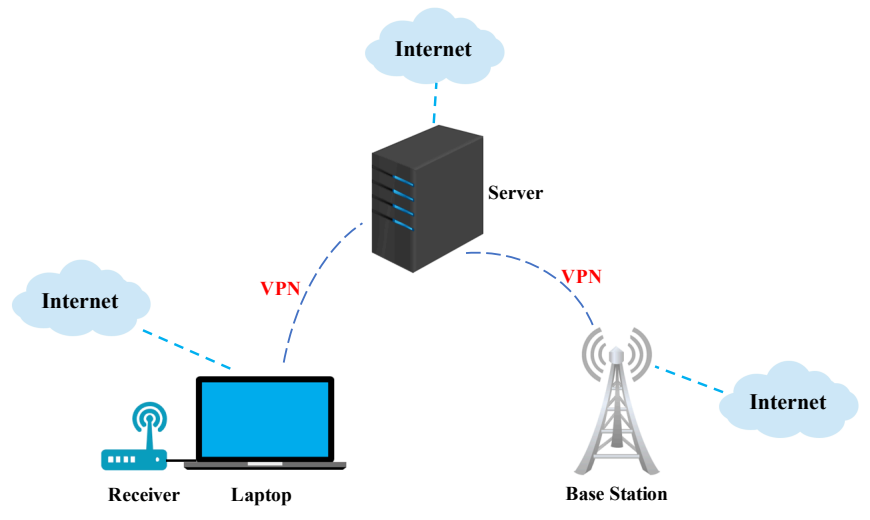

Fig. 3. Illustration of Network Connection.

using each receiver. One receiver was connected to the Internet using a local Wi-Fi system, while the other was connected to a $4 \mathrm{G}$ router. We carried out two sets of tests: static and dynamic tests.

Static tests were carried out at three different locations to examine different network profiles. Each experiment lasted for around two hours. The number of tracked GNSS satellites during each experiment is shown in Fig. 4. We can see that a variable number of 6 to 9 satellites were available. Fig. 5 and Fig. 6 plots localization errors and network time delays, respectively. In the first and third tests, the average latency of the Wi-Fi connection is much smaller than the latency of the $4 \mathrm{G}$ network. For the system using the Wi-Fi connection, the solutions converge faster to more accurate results compared to the system with the $4 \mathrm{G}$ connection. In the second test, the two networks have comparable latency. The latency of the Wi-Fi connection is only slightly smaller than that of the $4 \mathrm{G}$ network. The results based on the Wi-Fi connection are not as stable as test 1 and test 3. However, the Wi-Fi-based system still slightly outperforms the system relying on $4 \mathrm{G}$ access.

In the dynamic test, the platform moved on a rectangular

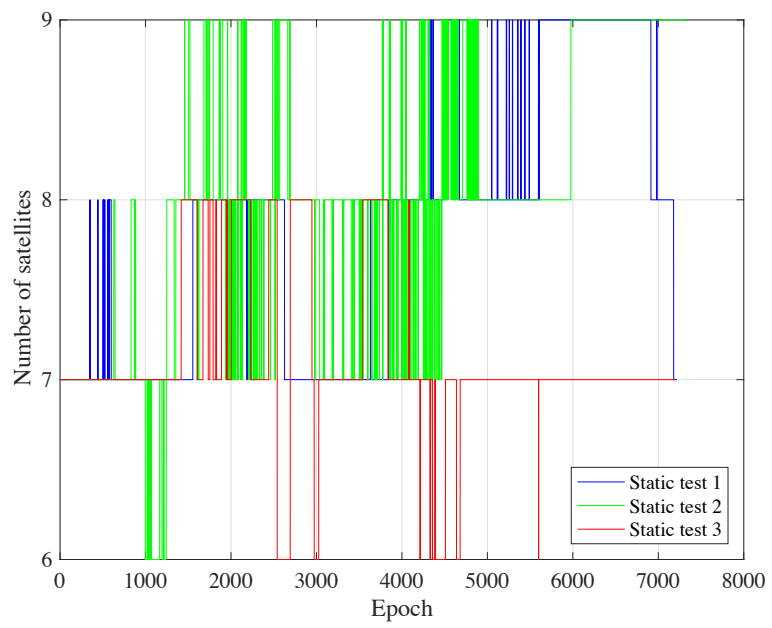

Fig. 4. Number of tracked GNSS satellites during the static experiments. 

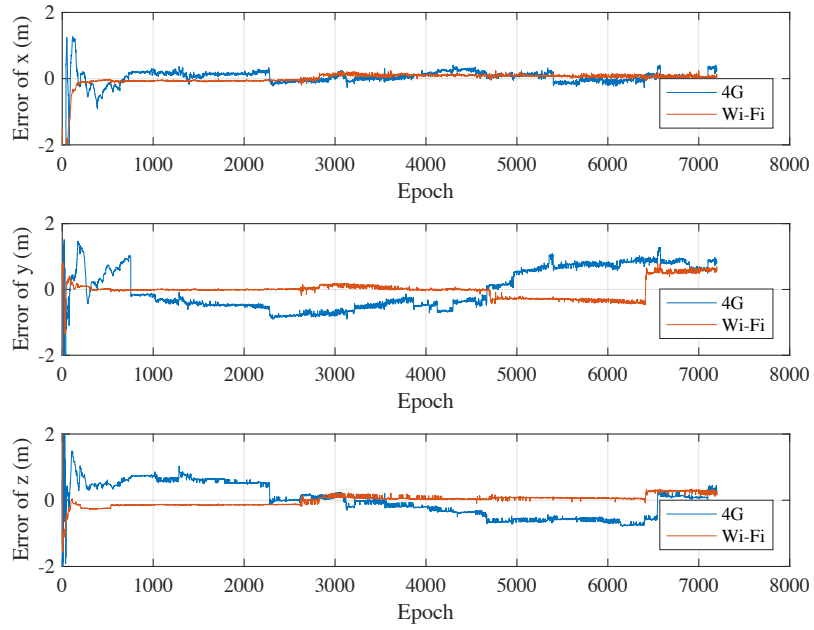

(a) Static test 1
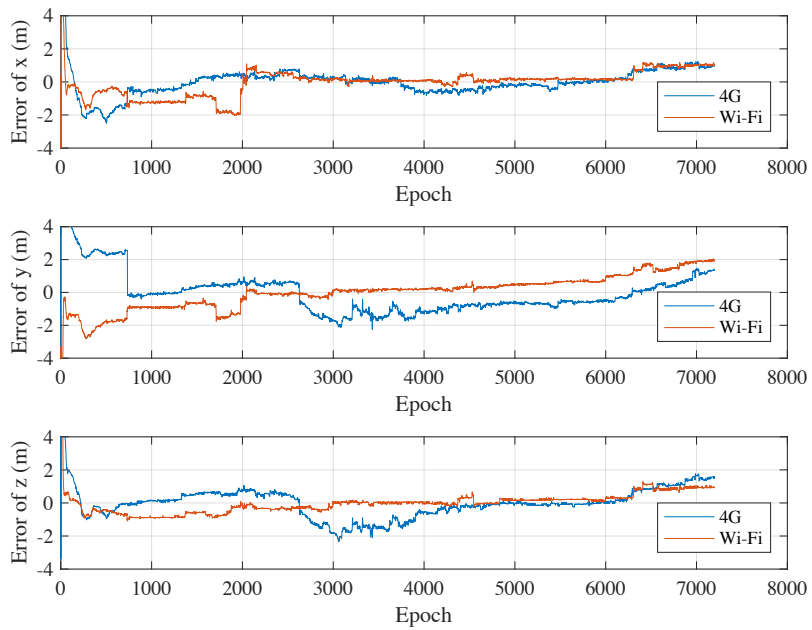

(b) Static test 2
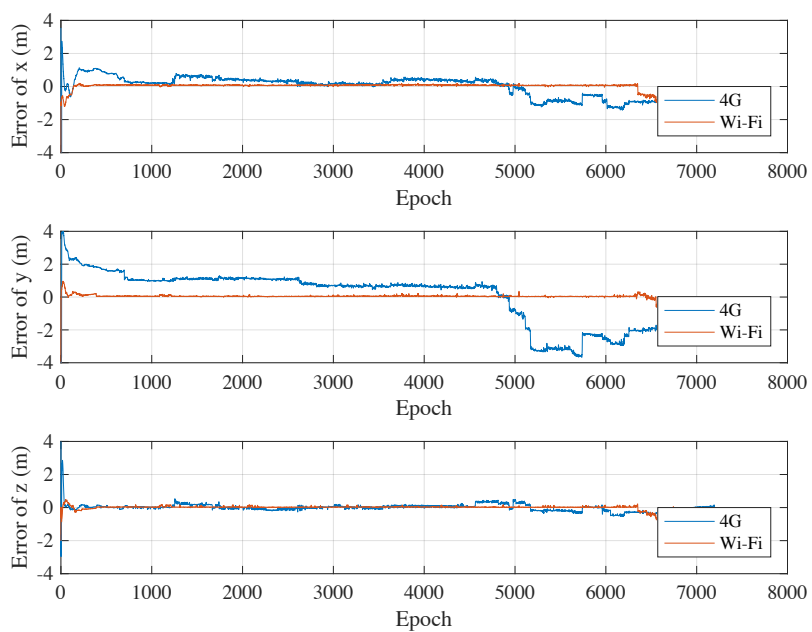

(c) Static test 3

Fig. 5. Positioning errors from three static experiments.

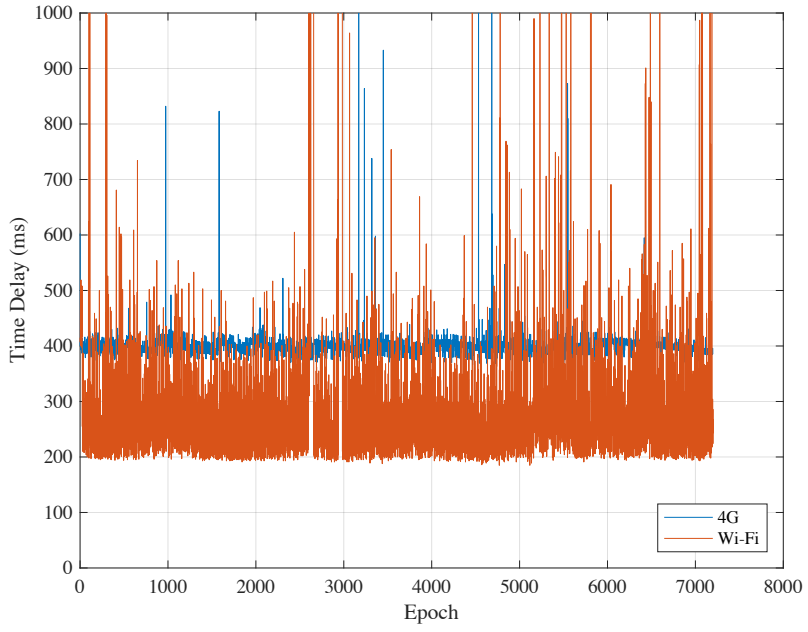

(a) Static test 1

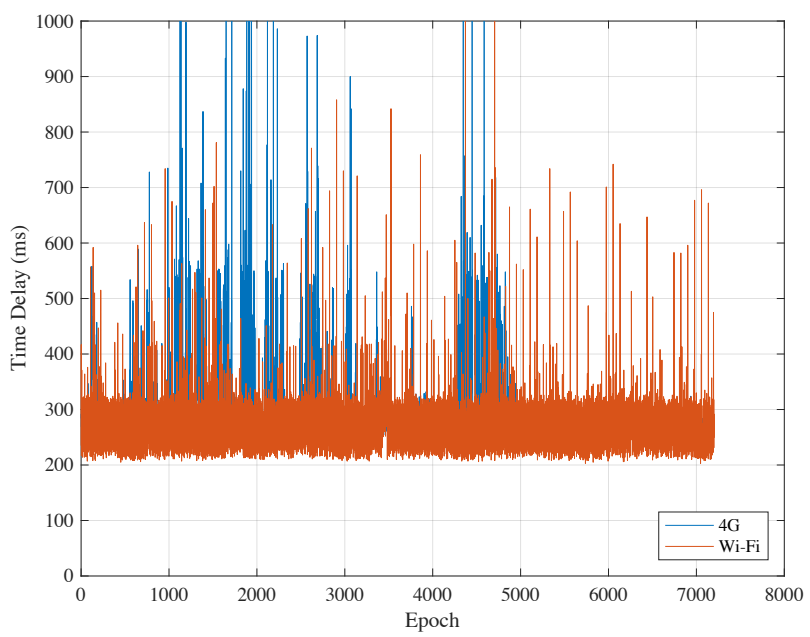

(b) Static test 2

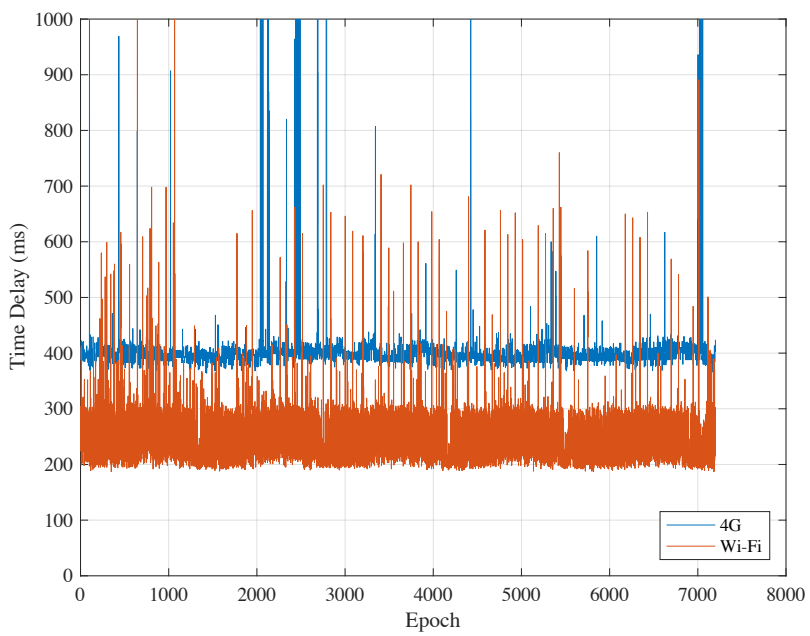

(c) Static test 3

Fig. 6. Network latency from three static experiments. 


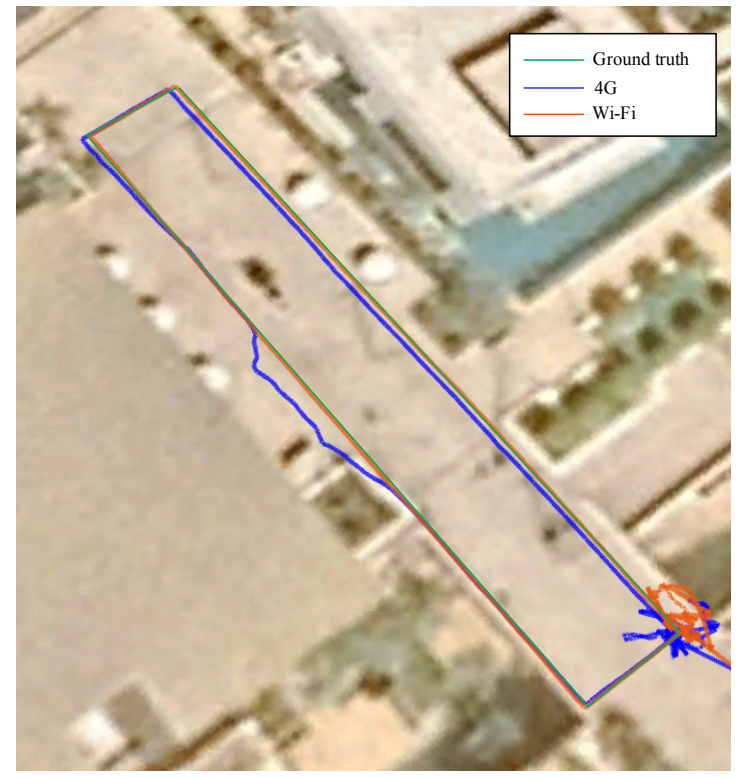

Fig. 7. Positioning solutions of dynamic experiment.

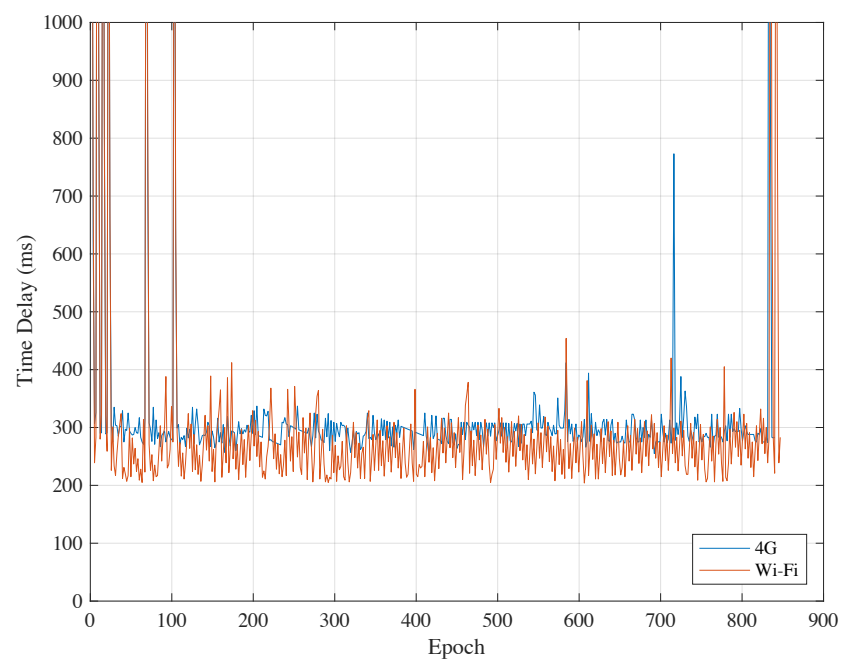

Fig. 8. Network latency for the dynamic experiment.

path. Initially, the platform was kept static at a corner of the path rectangle until RTK positioning converged for both receivers. The convergence times for $\mathrm{Wi}-\mathrm{Fi}$ and $4 \mathrm{G}$ were around 5 and 11 minutes, respectively. The experiment lasted in 14 minutes. A total of 9 GNSS satellites were observed during this period. Fig. 7 depicts the positioning solutions and the ground truth. The corresponding network latencies are shown in Fig. 8. From Fig. 7, it is evident that the results obtained from the system using the Wi-Fi connectivity are closer to the ground truth than those obtained from the system connecting to the $4 \mathrm{G}$ network. In addition, the Wi-Fi-based system shows significantly more stability, that its results do not occasionally drift away from the true path, as is the case for the $4 \mathrm{G}$ based system. This signifies that a small network latency has distinct advantages for both RTK convergence time and localization accuracy.

\section{CONCLUSIONS}

In this work, we use tinc-VPN software for network connectivity between a rover and a base station in the context of RTK positioning. The setup tested in this work enables secure connectivity without requiring the base station to have a fixed (public) IP address (for NTRIP access), which may not be achievable at a reasonable cost in remote outdoor locations. We provide a detailed performance evaluation under different network conditions. Experimental results demonstrate that small network latency can benefit RTK systems in both convergence time and positioning accuracy.

\section{REFERENCES}

[1] D. Slaughter, D. Giles, and D. Downey, "Autonomous robotic weed control systems: A review," Computers and electronics in agriculture, vol. 61, no. 1, pp. 63-78, 2008.

[2] D. Portugal, G. Cabrita, B. D. Gouveia, D. C. Santos, and J. A. Prado, "An autonomous all terrain robotic system for field demining missions," Robotics and Autonomous Systems, vol. 70, no. C, pp. 126-144, 2015.

[3] A. Parkins, "Increasing GNSS RTK availability with a new single-epoch batch partial ambiguity resolution algorithm," GPS solutions, vol. 15, no. 4, pp. 391-402, 2011.

[4] M. Bahrami and M. Ziebart, "Instantaneous doppler-aided RTK positioning with single frequency receivers," in IEEE/ION Position, Location and Navigation Symposium. IEEE, 2010, pp. 70-78.

[5] P. Misra and P. Enge, "Global Positioning System: signals, measurements and performance second edition," Massachusetts: Ganga-Jamuna Press 0, 0 (2006), pp. 466-490, 2006.

[6] C. Wang, Y. Feng, M. Higgins, and B. Cowie, "Assessment of commercial network RTK user positioning performance over long inter-station distances," Journal of Global Positioning Systems, vol. 9, no. 1, pp. 78-89, 2010.

[7] Y. Feng, J. Wang et al., "GPS RTK performance characteristics and analysis," Positioning, vol. 1, no. 13, 2008.

[8] T. Yan, S. Lim, and C. Rizos, "Performance analysis of real-time GNSS data distribution over the internet," Proceedings of SSC2009, Adelaide, Australia, vol. 28, pp. 491-502, 2009.

[9] V. Wegener and L. Wanninger, "Communication options for network RTK/SAPOS® realization," in Joint 2nd Workshop on Positioning, Navigation and Communication 2005 (WPNC '05) \& 1st Ultra-Wideband Expert Talk 2005 (UET '05). Citeseer, 2005.

[10] M. Uradzinski, D. Kim, and R. B. Langley, "The usefulness of internetbased (NTrip) RTK for navigation and intelligent transportation systems," in Proceedings of ION GNSS, 2008, pp. 6-19. 